ditions, respectively. CTLA-4 expression in MSC was analyzed on protein and on transcriptional levels by flow cytometry, immunoblotting, ELISA and by quantitative PCR, respectively. To assess the functionality of CTLA-4 expression, MSC were co-cultured with peripheral blood mononuclear cells (PBMC) isolated by density gradient centrifugation from venous blood of healthy donors. The cells were stimulated for $48 \mathrm{~h}$ with PHA $(5 \mu \mathrm{g} / \mathrm{ml})$ or left untreated and challenged using either CTLA4-Ig or anti-CTLA-4-antibody, incubated under normoxic $\left(\sim 18 \% \mathrm{O}_{2}\right)$ or hypoxic $\left(<1.5 \% \mathrm{O}_{2}\right)$ conditions and analyzed for TNF $\alpha$ secretion by suspension assay. Results: The MSC phenotype of bone-marrow derived cells could be verified according to their surface marker expression and their osteogenic and adipogenic differentiation capacity. On transcriptional level, MSC express both the full-length and - to a higher extent - the soluble CTLA-4 isoforms with a higher mRNA abundance under normoxic as compared to hypoxic conditions. Extra- and intracellular analysis of CTLA-4 expression on protein level, demonstrated a significant shift of the whole MSC population $(p<0,01)$. CTLA-4 expression and secretion by MSC was confirmed using immunoblot and ELISA, respectively. Co-culture of MSC with PHA-activated PBMC significantly reduced the amount of secreted TNF $\alpha(p<0,05)$ which could be reversed by anti-CTLA-4-antibody $(p<0,05)$, under both normoxic and compared to hypoxic conditions, respectively. Conclusions: We clearly demonstrate the existence of CTLA-4 on hMSC and its functionality with regard to the inhibition of PHA-induced TNF $\alpha$ secretion by PBMC. We also demonstrate that the expression of CTLA-4 (i) contributes to the immunomodulatory capacity of hMSC and (ii) supports the 'immune privileged' status of these cells.

Disclosure of Interest: None declared

DOI: 10.1136/annrheumdis-2017-eular.4078

\section{FRI0020 ALTERED BIOENERGETICS, MITOCHONDRIAL FUNCTION AND PRO-INFLAMMATORY PATHWAYS IN RA SYNOVIUM IN RESPONSE TO TOFACITINIB}

C. Orr ${ }^{1}$, T. Mcgarry $^{2}$, S. Wade ${ }^{3}$, M. Biniecka ${ }^{4}$, S. Wade ${ }^{5}$, D.J. Veale ${ }^{4}$, U. Fearon ${ }^{5}{ }^{1}$ Translational Rheumatology, St. Vincent's University Hospital; ${ }^{2}$ Clinical Medicine, Trinity Biomedical Sciences Institute; ${ }^{3}$ Molecular Rheumatology, Trinity Biomedical Sciences Centre; ${ }^{4}$ Center for Arthritis and Rheumatic Diseases, St Vincent's University Hospital; ${ }^{5}$ Molecular Rheumatology, Trinity Biomedical Sciences Center, Dublin, Ireland

Background: Rheumatoid arthritis (RA) is a chronic joint disease, characterised by synovial inflammation and a shift in the metabolic profile of cells to a more destructive phenotype. The JAK-STAT signalling pathway is implicated in the pathogenesis of RA.

Objectives: To examine the effect of tofacitinib, a selective JAK inhibitor, on synovial cellular bioenergetics, mitochondrial function and subsequent proinflammatory mechanisms in RA.

Methods: Ex-vivo RA whole tissue synovial explants and primary RA synovial fibroblasts (RASFC) and were cultured with tofacitinib $(1 \mu \mathrm{M})$ for $24-72 \mathrm{hrs}$. RASFC metabolism was assessed by the XF24-Flux-analyser and mitochondrial mutagenesis was quantified using a mitochondrial random mutation capture assay. Mitochondrial function was assessed for reactive oxygen species (ROS), mitochondrial membrane potential (MMP) and mitochondrial mass (MM) using the specific cell fluorescent probes and differential gene expression by mitochondrial gene arrays or RT-PCR. Mitochondrial structural morphology was assessed by transmission electron microscopy. Lipid peroxidation (4HNE) was measured by specific ELISA. Dual staining of pSTAT3 and mitochondrial marker Cox-IV was demonstrated by confocal microscopy. The effect of tofacitinib $(1 \mu \mathrm{M})$ in RA synovial explant on markers of cellular bioenergetics and pro-inflammatory mediators, including cytokines and growth factors were quantified by ELISA, MSD multiplex assays and Real-time PCR.

Results: An initial screen demonstrated alterations in 18 key genes involved in mitochondrial function in RA synovial tissue in response to tofacitinib. Supporting this, tofacitinib inhibited ROS production, decreased the MMP and MM (all $p<0.05$ ), coupled with altered mitochondrial morphology. No effect observed for mtDNA mutations or $4 \mathrm{HNE}$ levels. Tofacitinib significantly inhibited the expression of glycolytic genes HIF1 $\alpha$, HK2, LDHA, GSK3A and PDK1 (all $p<0.05)$ suggesting altered energy metabolism. This was paralleled by inhibition of baseline ECAR (glycolysis) with a concomitant increase in baseline OCR (oxidative phosphorylation), ATP production, maximal respiratory capacity and in the respiratory reserve in RASFC, confirming a bioenergetic switch in synovial cells in response to tofacitinib. Furthermore, we demonstrated co-localisation of pSTAT3 with Cox-IV in RASFC, suggested that in addition to nuclear transcription, PSTAT3 may also act as a mitoTF, regulating mitochondrial function directly. Finally, in RA whole tissue explants, tofacitinib significantly inhibited glycolytic genes HK2, GSK3A and PDK1 which was paralleled by a significant decrease in the spontaneous secretion of inflammatory mediators IL-6, IL-8, IL-1 $\beta$, ICAM-1, VEGF, Tie2 and MMP1 (all $p<0.05$ ).

Conclusions: In this study, we describe a potential mechanism of action for tofacitinib, through reversing mitochondrial dysfunction and subsequent switch in cellular bioenergetics, in favour of a less glycolytic microenvironment leading to the reduction of inflammatory mediators. Thus, we have demonstrated that pathological cellular metabolism may be reversed by therapeutic treatment with tofacitinib.

Disclosure of Interest: None declared
DOI: 10.1136/annrheumdis-2017-eular.6209

\section{FRI0021 INTRAVENOUS INFUSION OF FUCOSYLATED BONE MARROW MESENCHYME CELLS IN PATIENTS WITH OSTEOPOROSIS. PRECLINICAL STUDY IN A MURINE MODEL}

N. Lozano-Rivas ${ }^{1}$, V. Cabañas-Perianes ${ }^{2}$, L. Linares-Ferrando ${ }^{1}$, C. Marras Fernández-Cid ${ }^{1}$, M.D. Lucas López ${ }^{2}$, F.A. Martínez-Angosto ${ }^{1}$, M. Blanquer ${ }^{2}$, J. Martínez Ferrín ${ }^{1}$, A. García Hernández ${ }^{2}$, R. Sackstein ${ }^{3}$, J.M. Moraleda ${ }^{2}$. ${ }^{1}$ Rheumatology; ${ }^{2}$ Hematology, University Hospital Virgen de la Arrixaca, Murcia, Spain; ${ }^{3}$ Harvard Medical School, Cambridge, United States

Background: Osteoporosis is a skeletal disease characterized by systemic bone loss with an increased risk to bone fractures, associated with high morbidity and mortality ${ }^{1}$. Mesenchymal stem cells (MSC) from bone marrow (BM) are ideal candidates for the treatment of osteoporosis because they are able to differentiate to osteoblasts although its osteotropism is low ${ }^{2}$. Ex vivo fucosylation (adding a fucose residue) in $\alpha 1-3$ position of the CD44 antigen by use of the enzyme fucosyltransferase VI (FTVI) yields Hematopoietic cell E and L-selectin ligand (HCELL) in MSC increasing the affinity for E-selectin and osteotropism.

Objectives: To describe the safety and efficacy of human fucosylated MSC infused in an immunocompromised mice model (NOD/SCID).

Methods: $31 \mathrm{NOD} / \mathrm{SCID}$ mice were randomized to by tail vein injection: $1 \times 10^{6}$ fucosylated MSC $(n=13), 1 \times 10^{6}$ MSC $(n=14)$ or saline $(n=4)$. Toxicities were evaluated by a clinical score, weight and histological assessment (heart, lung, liver, spleen, kidney, gonads, brain, bone and BM). RT-PCR array-based evaluation of the expression of human $\beta$-actin and $\beta 2$-microglobulin genes was performed to study biodistribution of MSC. The maintenance of genetic integrity was evaluated during in vitro culture by karyotype. Additional samples of tibia and calota were to immunostained with a polyclonal Rabbit anti-human osteocalcina to demonstrate efficacy. Osteocalcin-positive cells were identified by a dark-brown cytoplasmic precipitate.

Results: There was no unexpected death and none of the mices had any acute toxicity. The histology of heart, liver, kidney, spleen, gonads, brain, bone and bone marrow was normal in all mice. There were localized areas of lung inflammation in $15 \%, 42 \%$ and $25 \%$ of mice infused with fucosylated MSC, MSC, and saline, respectively without significance differences $(p=0.28)$. Biodistribution was normal with except one mice (infused with MSC non-fucosylated) showed an expression of human RNA $\beta$-actin and $\beta 2$-microglobulin in the lung sample taken after 12 weeks of intravenous infusion. Osteoblasts were seen in 100\% of mices infused with fucosylated MSC, in $62.5 \% \%$ of animals infused with MSC alone, and none in saline group $(p=0.01)$. NOD/SCID mice infused with fucosylated MSC presented a higher number of osteoblasts positive for osteocalcin in 10 high-power fields (400x) of tibia and calvarium sections that non-fucosylated (32 vs 5.5) ( $p=0.0082$ ). Human osteoblasts were detected inside the bone from the fifth to the twelfth week after infusion.

Conclusions: Intravenous infusion of human fucosylated MSC is safe and effective in guiding the cells to bone with a higher potential to ossification in NOD/SCID mices that non-fucosylated MSC. These results are allowing to start a human clinical trial with intravenous fucosylated MSC as treatment in patients with osteoporosis.

\section{References:}

[1] Johnell O, Kanis JA. An estímate of the worldwide prevalence and disability associated with osteoporotic fractures.Osteoporos Int 2006 Dec;17(12):172633.

[2] Connolly JF. Injectable bone marrow preparations to stimulate osteogenic repair. Clin Orthop Relat Res 1995 Apr;(313):8-18.

Disclosure of Interest: None declared

DOI: 10.1136/annrheumdis-2017-eular.2945

\section{FRIDAY, 16 JUNE 2017}

\section{Rheumatoid arthritis - etiology, pathogenesis and animal models}

\section{FRI0022 GLUTAMINE METABOLISM PLAYS A KEY ROLE IN THE CELL GROWTH OF FIBROBLAST-LIKE SYNOVIOCYTES IN RHEUMATOID ARTHRITIS}

S. Takahashi, J. Saegusa, H. Yamada, Y. Ichise, I. Naka, T. Okano, K. Akashi, S. Sendo, Y. Ueda, A. Onishi, A. Morinobu. Rheumatology and Clinical Immunology, Kobe University Graduate School of Medicine, Kobe, Japan

Background: Many signaling pathways activated under inflammatory and hypoxic conditions have profound effects on intracellular metabolism to support cell growth and survival. The recent findings of cancer-specific metabolic changes, including increased glucose and glutamine consumption, has provided new therapeutic targets for consideration. The microenvironment in inflamed joints in RA is also characterized by hypoxia and low concentration of nutrients, and fibroblast-like synoviocytes from RA patients (RA-FLS) is known to have several tumor-like characteristics. However, the role of glucose and glutamine metabolism in the aberrant proliferation of these cells is unclear. 
Objectives: We evaluated the role of these metabolic pathways in RA-FLS proliferation and in autoimmune arthritis in SKG mice.

Methods: The expression of glycolysis- or glutaminolysis-related enzymes was evaluated by real-time PCR and Western blotting, and the intracellular metabolites were evaluated by metabolomic analyses. The effects of glucose or glutamine on RA-FLS cell growth were investigated using glucose- or glutamine-free medium. Glutaminase 1 (GLS1) siRNA and the GLS1 inhibitor compound 968 were used to inhibit GLS1 in RA-FLS. Arthritis was induced in SKG mice by zymosan A injection. Compound 968 was used to study the effect of GLS1 inhibition on Zymosan A-injected SKG mice. Ki-67-positive cells were analyzed by immunohistochemistry.

Results: GLS1 expression was increased in RA-FLS, and metabolomic analyses revealed that glutamine and glutamate consumption were increased in RA-FLS. RA-FLS proliferation was reduced under glutamine-deprived, but not glucosedeprived conditions. Cell growth of RA-FLS was inhibited by GLS1 siRNA transfection or GLS1 inhibitor treatment. Silencing of GLS1 in RA-FLS did not affect IL-6 or MMP-3 production in supernatants. GLS1 expression in RA-FLS was not affected by pro-inflammatory cytokine stimulation. Compound 968 ameliorated the autoimmune arthritis and decreased the number of Ki-67-positive synovial cells in SKG mice.

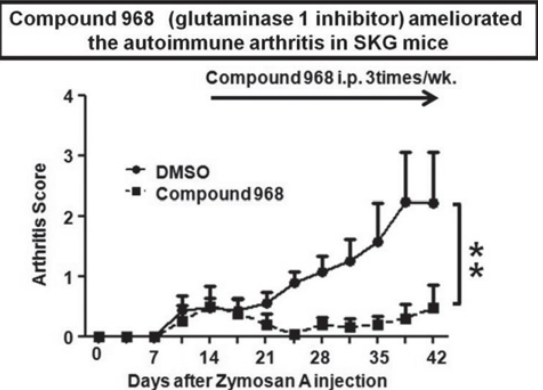

Conclusions: Our findings suggested that glutamine metabolism plays an important role in regulating RA-FLS proliferation, without being affected by proinflammatory cytokine stimulation or affecting cytokine production, and may be a novel therapeutic target for RA.

Disclosure of Interest: None declared

DOI: 10.1136/annrheumdis-2017-eular.2804

\section{FRI0023 ARTESUNATE CAN INHIBIT MIGRATION AND INVASION OF FIBROBLAST-LIKE SYNOVIOCYTES VIA SUPPRESSION OF MATRIX METALLOPROTEINASE 9 IN RHEUMATOID ARTHRITIS PATIENTS}

J. Jing ${ }^{1}$, T. Yan ${ }^{2}$, J.-D. Ma ${ }^{1}$, Y.-Q. Mo ${ }^{1}$, L. Dai ${ }^{1} .{ }^{1}$ Rheumatology, Sun Yat-sen Memorial Hospital, Sun Yat-sen University; ${ }^{2}$ Zhongshan School of Medicine, Sun Yat-Sen University, Guangzhou, China

Background: Evidences show that antimalarial agents of artemisinin and its derivatives such as artesunate may inhibit proinflammatory cytokines secretion from human rheumatoid arthritis (RA) fibroblast-like synoviocytes (FLS) in vitro. It has also been demonstrated that artesunate may ameliorate the symptoms of arthritis and prevent joint damage in collagen induced arthritis rat, which suggests that artesunate may be used for RA treatment. Recent studies show that RA-FLS is critical for joint destruction in RA because it can migrate and attach to cartilage and bone, and then invade them by secreting proteases such as matrix metalloproteinases (MMP) 9 in RA. However, effects of artesunate on migration and invasion of RA-FLS are poorly understood.

Objectives: To investigated the effects of artesunate on migration and invasion of RA-FLS and its underlying mechanism.

Methods: Synovial tissues were obtained from active RA patients as well as osteoarthritis (OA) and noninflammatory orthopedic arthropathies (Orth.A) patients and immumohistochemical (IHC) staining were performed for MMP9 expression. FLS isolated from these patients were analyzed for MMP9 exprssion by western blot (WB) and incubated with artesunate at different concentrations $(0 \mu \mathrm{M}, 20 \mu \mathrm{M}, 40 \mu \mathrm{M}$ and $60 \mu \mathrm{M})$, methotrexate (MTX, 10nM) or hydroxychloroquine

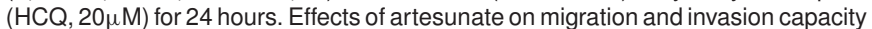
were detected by transwell and wound healing assays. MMP9 and PI3K/Akt signal transduction protein expression after artesunate treatment was measured by WB. Results: (1) IHC staining showed that synovial MMP9 expressed in lining and sublining area with intense nuclear and endochylema staining in RA synovium and the percentage of MMP9+ cells was significantly higher in RA $(n=32)$ than that in OA $(n=6)$ or Orth. A ( $n=6$, Figure $1 \mathrm{~A}, \mathrm{~B})$.

(2) Migration and wound healing assays for 12 hours and invasion assay for 24 hours showed that RA-FLS possessed stronger capacity in migration and invasion than OA-FLS or Orth.A-FLS (Figure 1E, F). Artesunate inhabits the migration and invasion capacity of RA-FLS in a dose-dependent manner. MTX also has an inhibition effect on the migration and invasion of RA-FLS, but not HCQ (Figure 2A).

(3) MMP9 expression in RA-FLS was significantly higher than that in OA-FLS or Orth.A-FLS (Figure 1C, D). $40 \mu \mathrm{M}$ or $60 \mu \mathrm{M}$ artesunate markedly inhibited the expression of MMP9 in RA-FLS by WB (Figure $2 \mathrm{~B}$ ).

(4) WB analysis showed artesunate suppressed generation of phophso-Akt in a dose-dependent manner which indicated that Akt activity (phophso-Akt/Akt) in $40 \mu \mathrm{M}$ and $60 \mu \mathrm{M}$ artesunate treatment groups were significantly lower than that in untreated group (Figure $2 \mathrm{C}$ ).

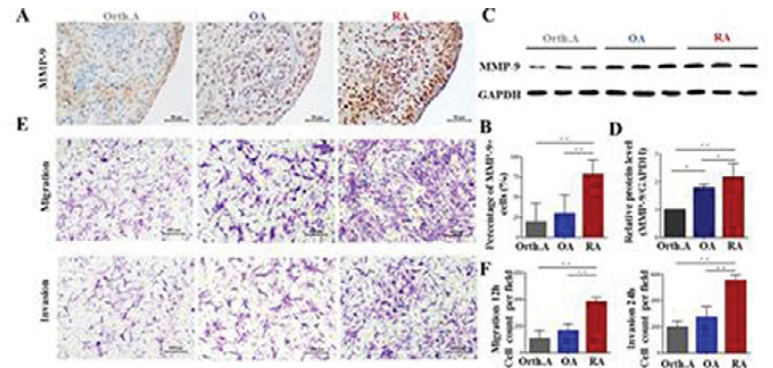

Figure 1 Expression of MMP9 in synovium and FLS and migration and invasion capacity of FLS. (A and B) IHC analysis showed that expression of MMP9 from RA patients was significantly higher than OA and Orth.A patients. Data are representative as means $\pm \mathrm{SD} * * P<0.01, * * * P<0.001$. (C and D) WB analysis showed that expression of MMP9 from RA-FLS was significantly higher than Orth.A-FLS or OA-FLS. Data were representative as means $\pm \mathrm{SD}, \bullet<<0.05, * P<0.01$. (E and $\mathbf{F}$ ) Transwell assays showed that RA-FLS possess stronger capacity of migration and invasion than OA-FLS or Orth.A-FLS. Data were representative as means $\pm \mathrm{SD}, * * P<0.01$.

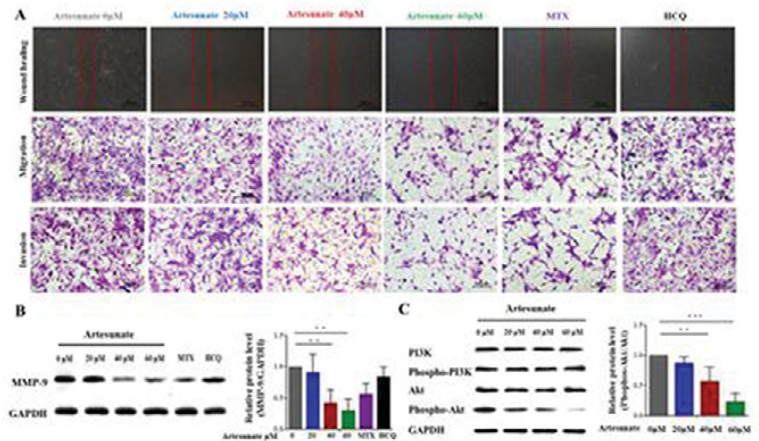

Figure 2 Effects of artesunate on the migration and invasion capacity and MMP9, PI3k/Akt pathway expression. (A) Artesunate inhabits the migration and invasion capacity of RA-FLS in a dose-dependent manner. (B) WB analysis of RA-FLS showed that MMP9 expression was siginificantly inhibited with treatment with $40 \mu \mathrm{M}$ and $60 \mu \mathrm{M}$ artesunate. (C) WB analysis showed that artesunate suppressed the expression of phophso-Akt in a dose-dependent manner. Bar are representative as means $\pm \mathrm{SD}, * P<0.01$, $\cdots p<0.001$

Conclusions: Artesunate could inhibit the migration and invasion capacity of RA-FLS and the expression of MMP9 through suppressing Akt activity.

Acknowledgements: This work was supported by National Natural Science Foundation of China (81671612 and 81471597), Research Project of Traditional Chinese Medicine Bureau of Guangdong Province (20161058) and Guangdong Natural Science Foundation (2014A030313074).

Disclosure of Interest: None declared

DOI: 10.1136/annrheumdis-2017-eular.2039

\section{FRI0024 MICRORNA-146A CONTROLS LOCAL BONE DESTRUCTION BY REGULATING FIBROBLAST INDUCED OSTEOCLASTOGENESIS IN INFLAMMATORY ARTHRITIS}

V. Saferding ${ }^{1}$, A. Puchner ${ }^{1}$, E. Goncalves-Alves ${ }^{1}$, M. Hofmann ${ }^{1}$, M. Bonelli ${ }^{1}$, S. Hayer ${ }^{1}$, H.P. Kiener ${ }^{1}$, P. Georgel ${ }^{2}$, M.I. Koenders ${ }^{3}$, G. Steiner ${ }^{1}$,

J.S. Smolen ${ }^{1}$, K. Redlich ${ }^{1}$, S. Blueml ${ }^{1}$. ${ }^{1}$ Medical University of Vienna, Vienna, Austria; ${ }^{2}$ Université de Strasbourg, Strasbourg, France; ${ }^{3}$ Radboud University Nijmegen, Nijmegen, Netherlands

Background: MicroRNA (MiR-) 146a plays an important role in the regulation of the innate immune response and has also been shown to suppress cancer development in myeloid cells. Although in late stages of arthritis elevated expression of miR-146a in synovial tissue of rheumatoid arthritis patients was detected, the level of this miRNA was found to be down regulated in early disease, but its role in the development of inflammatory arthritis is still elusive.

Objectives: The objective of this study is to analyse the role of miR-146a in arthritis by the use of a chronic arthritis disease model. We aim to investigate the regulatory function of this miRNA in the pathogenic stroma, therefore in fibroblasts but also in immune cells.

Methods: To induce arthritis we used the chronic inflammatory hTNFtg disease model, therefore we crossed miR-146a deficient into hTNFtg mice. Disease severity was assessed clinically and histologically. Blood of arthritis animals was analysed by flow cytometry. Serum cytokine levels were measured by Elisa. Synovial fibroblasts were isolated from metatarsal bones and their proliferation 